INPLASY

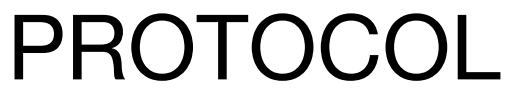

To cite: Li et al. Different Neoadjuvant Treatment Regimens of Early Triple Negative Breast Cancer: A Systematic Review and Network Meta-analysis. Inplasy protocol 2020110114. doi:

10.37766/inplasy2020.11.0114

Received: 26 November 2020

Published: 26 November 2020

Corresponding author: Junjie Li

lijunjie_ronaldo@hotmail.com

Author Affiliation:

Shanghai Medical College, Fudan University

Support: Sanofi funding.

Review Stage at time of this submission: Formal screening of search results against eligibility criteria.

\section{Different Neoadjuvant Treatment Regimens of Early Triple Negative Breast Cancer: A Systematic Review and Network Meta-analysis}

\author{
Li, JJ1; Chen, L2; Wang, ZH³; Shao, ZM4.
}

Review question / Objective: To explore the efficacy and safety of neoadjuvant treatment for patients with early or locally advanced human epidermal growth factor receptor 2 negative (HER2-) and hormone receptor negative (HR-) breast cancer (BC).

Condition being studied: Early or locally advanced triple negative breast cancer.

Information sources: A systematic search in the databases of Medline, EMBASE, Cochrane Library and Web of Science will be conducted in literatures published from inception to September $\mathbf{2 0 2 0}$ without limitations on date/time, language, or document type. Reference lists of included studies will be checked for relevant studies to identify any additional published or unpublished material (grey literature) not retrieved by the electronic search. Search strategies for all databases will be described in detail.

INPLASY registration number: This protocol was registered with the International Platform of Registered Systematic Review and Meta-Analysis Protocols (INPLASY) on 26 November 2020 and was last updated on 26 November 2020 (registration number INPLASY2020110114).

Conflicts of interest:

None.

\section{INTRODUCTION}

Review question / Objective: To explore the efficacy and safety of neoadjuvant treatment for patients with early or locally advanced human epidermal growth factor receptor 2 negative (HER2-) and hormone receptor negative (HR-) breast cancer (BC).
Condition being studied: Early or locally advanced triple negative breast cancer

\section{METHODS}

Participant or population: Patients with early or locally advanced HER2 negative and HR negative BC. HR- defined as both estrogen negative (ER-) and progesterone 
negative (PR-). The stage of the patients proven to be early or locally advanced, clinical stage of I to III or MO. There will be no limitation on age, sex or co-morbidity. Patients in studies with subgroup analysis of triple negative $\mathrm{BC}$ will be also included only if these patients were stratified by the factor of receptor status when randomized.

Intervention: Neoadjuvant treatment regimens that included drugs listed below: Paclitaxel; Docetaxel; Platinum: platinum/ cisplatin/carboplatin/oxaliplatin; $\mathrm{Nab}$ paclitaxel; Fluorouracil (capecitabine) ge m citabine / 5 - fl u orouracil) ; Anthracyclines (doxorubicin/ epirubicin); Cyclophosphamide; PD 1/PD L1 (pembrolizumab/Nivolumab/Atezolizumab); PARPi inhibitor (valiparib/olaparib); Everolimus. We will include single or a combination of any of the above-listed drugs used as neoadjuvant treatment. Concurrent or sequential chemotherapy will be included. There will be no limitation on dosage, frequency, time, method of administration, treatment duration, other combined therapy or line of treatment.

Comparator: Neoadjuvant treatment regimens that included drugs listed below: Paclitaxel; Docetaxel; Platinum: platinum/ cisplatin/carboplatin/oxaliplatin; $\mathrm{Nab}$ paclitaxel; Fluorouracil (capecitabine/ gem citabine / 5 - fluorouracil); Anthracyclines (doxorubicin/ epirubicin); Cyclophosphamide; PD 1/PD L1 (pembrolizumab/Nivolumab/Atezolizumab); PARPi inhibitor (valiparib/olaparib); Everolimus. We will include single or a combination of any of the above-listed drugs used as neoadjuvant treatment. Concurrent or sequential chemotherapy will be included. There will be no limitation on dosage, frequency, time, method of administration, treatment duration, other combined therapy or line of treatment.

Study designs to be included: Randomized controlled trials.

Eligibility criteria: Exclusion criteria: We will exclude literatures not reported in English or Chinese. We will exclude literatures only available with abstract, without detail reports We will exclude literatures which did not report pCR or serious AEs (Grade 3-5 AEs). If multiple publications were reported for the same trial or included the same or overlapping patient groups, only publications with the longest follow up or with largest sample size will be included.

Information sources: A systematic search in the databases of Medline, EMBASE, Cochrane Library and Web of Science will be conducted in literatures published from inception to September 2020 without limitations on date/time, language, or document type. Reference lists of included studies will be checked for relevant studies to identify any additional published or unpublished material (grey literature) not retrieved by the electronic search. Search strategies for all databases will be described in detail.

Main outcome(s): Pathologic complete remission ( $p C R$ ), fulfilling one of the following definitions or other common clinical definitions: no residual invasive tumor in breast and axillary lymph nodes, after neoadjuvant treatment (ypTO/is ypNO); no residual invasive or non-invasive tumor in breast and axillary lymph nodes, after neoadjuvant treatment (ypTO ypNO) Safety outcomes will include: Incidence of serious AEs (Grade 3-5 AEs) We will collect the outcome data measured at the longest follow-up unless otherwise specified. There will be no limitation on publication year, publication status, duration of study followup or period of study conduct.

Data management: For each study, the following information will be extracted into Excel by two reviewers (LJJ and CL) independently: the first author's name, the published year, country, center, diagnosis, diagnostic criteria, clinical stage, inclusion criteria, exclusion criteria, sample sizes, sex and age of patients, breast cancer susceptibility gene mutation status, baseline Eastern Cooperative Oncology Group performance status, time of followup, intervention, dosage of intervention and effect sizes of intervention. Disagreements will be resolved by discussion, with assistance from a third party if necessary. 
Quality assessment / Risk of bias analysis: Two reviewers (LJJ and WZH) will independently assess the risk of bias in the included studies. We will evaluate every domain of risk of bias, on the basis of the standard criteria outlined by the Cochrane Collaboration, including sequence generation, allocation concealment, blinding of participants and personnel, blinding of outcome assessment, incomplete outcome data, selective outcome reporting, and other bias. Disagreements will be resolved by discussion, with assistance from a third party if necessary.

Strategy of data synthesis: A Bayesian network meta-analysis will be performed by $R$ software. The fixed effect models will be used. The pooled estimation and the probability of which drug is the best will be obtained by the Markov Chains Monte Carlo method. The model convergence will be assessed by trace plots and BrooksGelman-Rubin plots [5]. The results of dichotomous outcomes will be reported as posterior medians of odds ratio (OR) and its credible intervals (Crls). Evidence inconsistency and clinical similarity in patient characteristics and settings across trials will be carefully assessed. Network geometry will be performed by STATA software. Network geometry will use nodes to represent different interventions and edges to represent the head-to-head comparisons between interventions. The size of nodes and thickness of edges will be associated with numbers of interventions and numbers of included trials, respectively.

\section{Subgroup analysis: None.}

Sensibility analysis: None.

Language: No language limitation on the search. Records with language other than in English or Chinese will be excluded when screening.

Country(ies) involved: China.

Keywords: Breast cancer; triple negative; neoadjuvant; network meta-analysis.
Contributions of each author:

Author 1 - Junjie Li - The author drafted and revised the protocol.

Email: lijunjie_ronaldo@hotmail.com

Author 2 - Li Chen - The author drafted the protocol.

Email: chen_li@fudan.edu.cn

Author 3 - Zhonghua Wang - The author drafted the protocol.

Email: zhonghuawang95@hotmail.com Author 4 - Zhimin Shao - The author drafted the protocol.

Email: zhimingshao@yahoo.com 